\title{
ON CURVES LYING ON A RATIONAL NORMAL SURFACE SCROLL
}

\author{
WANSEOK LEE AND EUISUNG PARK
}

\begin{abstract}
In this paper, we study the minimal free resolution of non-ACM divisors $X$ of a smooth rational normal surface scroll $S=S\left(a_{1}, a_{2}\right) \subset \mathbb{P}^{r}$. Our main result shows that for $a_{2} \geq 2 a_{1}-1$, there exists a nice decomposition of the Betti table of $X$ as a sum of much simpler Betti tables. As a by-product of our results, we obtain a complete description of the graded Betti numbers of $X$ for the cases where $S=S(1, r-2)$ for all $r \geq 3$ and $S=S(2, r-3)$ for all $r \geq 6$.
\end{abstract}

\section{INTRODUCTION}

Let $X \subset \mathbb{P}^{r}$ be a nondegenerate projective subvariety defined over an algebraically closed field $\mathbb{k}$. Various interesting properties of $X$ can be obtained from the minimal graded free resolution of its homogeneous vanishing ideal. But there are only a few cases where the free resolution is completely known.

The purpose of this paper is to study the minimal free resolution of $X$ when it is a curve lying on a smooth rational normal surface scroll.

Recall that if $S \subset \mathbb{P}^{r}$ is a nondegenerate projective surface then its degree is at least $r-1$, and $S$ is called a surface of minimal degree when $\operatorname{deg}(S)=r-1$. It is well-known that $S$ is either a quadric of rank $=4$ or the Veronese surface in $\mathbb{P}^{5}$ or a rational normal surface scroll (cf. [EH]). There have been several results which show that projective curves that are contained in a surface of minimal degree behave extremally with respect to various properties. More precisely, let $X \subset \mathbb{P}^{r}$ be a nondegenerate projective integral curve of degree $d$. Let $R:=\mathbb{k}\left[x_{0}, \ldots, x_{r}\right]$ be the homogeneous coordinate ring of $\mathbb{P}^{r}$ and $I(X)$ the defining ideal of $X$. The graded Betti numbers of $X$ are defined by

$$
\beta_{i, j}(X):=\operatorname{dim}_{\mathbb{k}} \operatorname{Tor}_{i}^{R}(I(X), \mathbb{k})_{i+j}
$$

and the Betti table of $X$, denoted by $\beta(X)$, is the table whose entry in the $i$-th column and $j$-th row is $\beta_{i, j}(X)$. Throughout this paper, we present $\beta(X)$ as follows:

$\beta(X)=$\begin{tabular}{|c||c|c|c|c|c|c|c|}
\hline$i$ & 0 & 1 & $\cdots$ & $i$ & $\cdots$ & $r-1$ & $r$ \\
\hline$\vdots$ & $\vdots$ & $\vdots$ & $\ddots$ & $\vdots$ & $\ddots$ & $\vdots$ & $\vdots$ \\
\hline$\beta_{i, 3}$ & $\beta_{0,3}(X)$ & $\beta_{1,3}(X)$ & $\cdots$ & $\beta_{i, 3}(X)$ & $\cdots$ & $\beta_{r-1,3}(X)$ & $\beta_{r, 3}(X)$ \\
\hline$\beta_{i, 2}$ & $\beta_{0,2}(X)$ & $\beta_{1,2}(X)$ & $\cdots$ & $\beta_{i, 2}(X)$ & $\cdots$ & $\beta_{r-1,2}(X)$ & $\beta_{r, 2}(X)$ \\
\hline
\end{tabular}

For example, $\beta_{0,2}(X)$ is the number of quadratic generators of $I(X)$.

Date: Busan, Seoul, December 5, 2018.

2010 Mathematics Subject Classification. 13D02, 14J26, 14 N05.

Key words and phrases. Minimal free resolution, Rational normal surface scroll, Divisor. 
The classical Castelnuovo Lemma shows that if $d \geq 2 r+1$, then $\beta_{0,2}(X) \leq\left(\begin{array}{c}r-1 \\ 2\end{array}\right)$ and equality is attained if and only if $X$ lies on a surface of minimal degree. G. Castelnuovo gave an upper bound of the arithmetic genus of $X$ and proved that his bound is achieved only if $X$ lies on a surface of minimal degree (cf. [H]). M. Green's $K_{p, 1}$ Theorem in G] says that $\beta_{i, 2}(X)=0$ if $i \geq r-1, \beta_{r-2,2}(X) \neq 0$ if and only if $X$ is a rational normal curve, and $\beta_{r-3,2}(X) \neq 0$ if and only if $X$ lies on a surface of minimal degree. Also, it is proved in $[\mathrm{MV}]$ and $[\mathrm{M}]$ that if $X$ is a $k$-Buchsbaum curve then

$$
\operatorname{reg}(X) \leq\left\lceil\frac{d-1}{r-1}\right\rceil+\max \{k, 1\}
$$

and when $k>0$ and $d \geq 2 r^{2}-3 r+3$, the equality $\operatorname{reg}(X)=\left\lceil\frac{d-1}{r-1}\right\rceil+k$ holds only if $X$ lies on a surface of minimal degree.

The above results lead our attention to the problem of studying the minimal free resolution of $X$ when it is a curve contained in a surface $S$ of minimal degree. In [N, Theorem 2.4], U. Nagel obtains a complete description of $\beta(X)$ when $\mathrm{X}$ is arithmetically CohenMacaulay. Note that $X$ is always ACM if $S$ is the Veronese surface in $\mathbb{P}^{5}$ or a singular rational normal surface scroll (cf. [N, Proposition 2.9] and [Fe, Example 5.2]).

Now, let $S=S\left(a_{1}, a_{2}\right)$ be a smooth rational normal scroll in $\mathbb{P}^{r}$ such that $1 \leq a_{1} \leq a_{2}$ and $r=a_{1}+a_{2}+1$. Thus the divisor class group of $S$ is freely generated by the hyperplane section $H$ and a ruling line $F$ of $S$. When $X$ is linearly equivalent to $a H+b F$, it is nondegenerate in $\mathbb{P}^{r}$ if and only if

$$
\text { either } \quad a=0 \text { and } b>a_{2} \quad \text { or } \quad a=1 \text { and } b \geq 1 \quad \text { or } \quad a \geq 2 \text { and } b \geq-a a_{2}
$$

(cf. [P2, Lemma 2.2]). Concerned with the minimal free resolution of $X$, it is an interesting and important property that $\beta(X)$ is invariant inside the divisor class of $X$. That is, if $X^{\prime}$ is a curve in $S$ and $X^{\prime} \equiv X$, then $\beta(X)=\beta\left(X^{\prime}\right)$ (cf. [P2, Proposition 4.1]). Finally, note that the graded Betti numbers of $X$ are completely known when $a \geq 1$ and $b \leq 1$ (cf. [P2, Theorem 4.3 and Theorem 4.4]). Along this line, a more precise goal of this paper is to study the following problem.

Problem ( $\dagger$ ). Let $S$ and $X$ be as above such that

$$
\text { either } \quad a=0 \text { and } b>a_{2} \text { or } \quad a \geq 1 \text { and } b \geq 2 \text {. }
$$

Then describe $\beta(X)$ completely (in terms of the integers $a_{1}, a_{2}, a$ and $b$ ).

The first general result associated with this problem is

Theorem 1.1 (Theorem 4.8 in [GM]). Let $X$ be an effective divisor of the smooth quadric $S=S(1,1)$ in $\mathbb{P}^{3}$ which is linearly equivalent to $a H+b F$ where $a \geq 0$ and $b \geq 2$. Then 


$\beta(X)=$\begin{tabular}{|c||c|c|c|}
\hline$i$ & 0 & 1 & 2 \\
\hline$\beta_{i, a+b}$ & $b+1$ & $2 b$ & $b-1$ \\
\hline$\beta_{i, a+b-1}$ & 0 & 0 & 0 \\
\hline$\vdots$ & $\vdots$ & $\vdots$ & $\vdots$ \\
\hline$\beta_{i, 3}$ & 0 & 0 & 0 \\
\hline$\beta_{i, 2}$ & 1 & 0 & 0 \\
\hline
\end{tabular}

In this paper, we extend Theorem 1.1 to all $S$ and $X$ that satisfy the following conditions (1.2) :

$$
a_{2} \geq 2 a_{1}-1 \quad \text { and } \quad b \equiv \epsilon\left(\bmod a_{2}\right) \quad \text { for some } a_{1}+1 \leq \epsilon \leq a_{2}+1
$$

To be more precise, Theorem 3.2 says that if $S$ and $X$ satisfy (1.2), then $\beta(X)$ is expressed as the sum of several Betti tables that are much simpler. Also in Propositions 4.1 and its corollaries, we obtain a complete description of those simpler Betti diagrams that make up $\beta(X)$.

When $a_{1}=1$ and hence $a_{2}=r-2$ for all $r \geq 3$, every $X$ considered in Problem $(\dagger)$ satisfies the conditions in (1.2). So, using Theorem 3.2 and Propositions 4.1, we solve Problem $(\dagger)$ entirely in case of $a_{1}=1$. For details, see Theorem 5.1. In particular, our results reprove Theorem 1.1. The following two theorems are obtained by applying Theorem 5.1 to the cases where $S=S(1,2)$ and $S=S(1,3)$, respectively. These results and their proofs illustrate how the main results of this paper can be applied in specific cases.

Theorem 1.2. Let $X$ be an effective divisor of $S=S(1,2)$ in $\mathbb{P}^{4}$ linearly equivalent to $a H+b F$ where either $a=0$ and $b \geq 3$ or else $a \geq 1$ and $b \geq 2$. Then $\beta(X)$ is equal to the first (resp. the second) one of the following two tables in Table 1 when $b=2 \delta$ (resp. $b=2 \delta+1)$ :

\begin{tabular}{|c||c|c|c|c|}
\hline$\beta_{i, a+b}$ & 1 & 3 & 3 & 1 \\
\hline$\vdots$ & $\vdots$ & $\vdots$ & $\vdots$ & $\vdots$ \\
\hline$\beta_{i, a+\delta+2}$ & 1 & 3 & 3 & 1 \\
\hline$\beta_{i, a+\delta+1}$ & 1 & 6 & 5 & 1 \\
\hline$\beta_{i, a+\delta}$ & 1 & 0 & 0 & 0 \\
\hline$\vdots$ & $\vdots$ & $\vdots$ & $\vdots$ & $\vdots$ \\
\hline$\beta_{i, 3}$ & 0 & 0 & 0 & 0 \\
\hline$\beta_{i, 2}$ & 3 & 2 & 0 & 0 \\
\hline
\end{tabular}

\begin{tabular}{|c||c|c|c|c|}
\hline$\beta_{i, a+b}$ & 1 & 3 & 3 & 1 \\
\hline$\vdots$ & $\vdots$ & $\vdots$ & $\vdots$ & $\vdots$ \\
\hline$\beta_{i, a+\delta+2}$ & 1 & 3 & 3 & 1 \\
\hline$\beta_{i, a+\delta+1}$ & 3 & 6 & 3 & 0 \\
\hline$\beta_{i, a+\delta}$ & 0 & 0 & 0 & 0 \\
\hline$\vdots$ & $\vdots$ & $\vdots$ & $\vdots$ & $\vdots$ \\
\hline$\beta_{i, 3}$ & 0 & 0 & 0 & 0 \\
\hline$\beta_{i, 2}$ & 3 & 2 & 0 & 0 \\
\hline
\end{tabular}

TABLE 1. $X \subset S(1,2)$ where $b=2 \delta$ and $b=2 \delta+1$, respectively.

Here, the Betti numbers lying in the vertical dots on Table 1 are as follows:

\begin{tabular}{|c|c|c|c|c|c|}
\hline$\overline{\beta_{i, a+j}}$ & 1 & 3 & $\overline{3}$ & 3 & for $\delta+2 \leq j \leq b$ and \\
\hline$\beta_{i, k}$ & 0 & 0 & & 0 & \\
\hline
\end{tabular}


Theorem 1.3. Let $X$ be an effective divisor of $S=S(1,3)$ in $\mathbb{P}^{5}$ linearly equivalent to $a H+b F$ where either $a=0$ and $b \geq 4$ or else $a \geq 1$ and $b \geq 2$. Then $\beta(X)$ is equal to the first (resp. the second and the third) one of the following three tables in Table 2 when $b=3 \delta-1$ (resp. $b=3 \delta$ and $b=3 \delta+1)$ :

\begin{tabular}{|c||c|c|c|c|c|}
\hline$\beta_{i, a+b}$ & 1 & 4 & 6 & 4 & 1 \\
\hline$\beta_{i, a+b-1}$ & 0 & 0 & 0 & 0 & 0 \\
\hline$\vdots$ & $\vdots$ & $\vdots$ & $\vdots$ & $\vdots$ & $\vdots$ \\
\hline$\beta_{i, a+\delta+3}$ & 1 & 4 & 6 & 4 & 1 \\
\hline$\beta_{i, a+\delta+2}$ & 0 & 0 & 0 & 0 & 0 \\
\hline$\beta_{i, a+\delta+1}$ & 1 & 4 & 10 & 6 & 1 \\
\hline$\beta_{i, a+\delta}$ & 2 & 4 & 0 & 0 & 0 \\
\hline$\vdots$ & $\vdots$ & $\vdots$ & $\vdots$ & $\vdots$ & $\vdots$ \\
\hline$\beta_{i, 3}$ & 0 & 0 & 0 & 0 & 0 \\
\hline$\beta_{i, 2}$ & 6 & 8 & 3 & 0 & 0 \\
\hline
\end{tabular}

\begin{tabular}{|c||c|c|c|c|c|}
\hline$\beta_{i, a+b}$ & 1 & 4 & 6 & 4 & 1 \\
\hline$\beta_{i, a+b-1}$ & 0 & 0 & 0 & 0 & 0 \\
\hline$\vdots$ & $\vdots$ & $\vdots$ & $\vdots$ & $\vdots$ & $\vdots$ \\
\hline$\beta_{i, a+\delta+3}$ & 0 & 0 & 0 & 0 & 0 \\
\hline$\beta_{i, a+\delta+2}$ & 1 & 4 & 6 & 4 & 1 \\
\hline$\beta_{i, a+\delta+1}$ & 0 & 6 & 8 & 3 & 0 \\
\hline$\beta_{i, a+\delta}$ & 1 & 0 & 0 & 0 & 0 \\
\hline$\vdots$ & $\vdots$ & $\vdots$ & $\vdots$ & $\vdots$ & $\vdots$ \\
\hline$\beta_{i, 3}$ & 0 & 0 & 0 & 0 & 0 \\
\hline$\beta_{i, 2}$ & 6 & 8 & 3 & 0 & 0 \\
\hline
\end{tabular}

\begin{tabular}{|c||c|c|c|c|c|}
\hline$\beta_{i, a+b}$ & 1 & 4 & 6 & 4 & 1 \\
\hline$\beta_{i, a+b-1}$ & 0 & 0 & 0 & 0 & 0 \\
\hline$\vdots$ & $\vdots$ & $\vdots$ & $\vdots$ & $\vdots$ & $\vdots$ \\
\hline$\beta_{i, a+\delta+3}$ & 1 & 4 & 6 & 4 & 1 \\
\hline$\beta_{i, a+\delta+2}$ & 0 & 0 & 0 & 0 & 0 \\
\hline$\beta_{i, a+\delta+1}$ & 4 & 12 & 12 & 4 & 0 \\
\hline$\beta_{i, a+\delta}$ & 0 & 0 & 0 & 0 & 0 \\
\hline$\vdots$ & $\vdots$ & $\vdots$ & $\vdots$ & $\vdots$ & $\vdots$ \\
\hline$\beta_{i, 3}$ & 0 & 0 & 0 & 0 & 0 \\
\hline$\beta_{i, 2}$ & 6 & 8 & 3 & 0 & 0 \\
\hline
\end{tabular}

TABLE 2. $X \subset S(1,3)$ where $b=3 \delta-1, b=3 \delta$ and $b=3 \delta+1$, respectively.

Here, the Betti numbers lying in the vertical dots table 2 are as follows:

\begin{tabular}{|c|c|c|c|c|c|c|}
\hline$\beta_{i, a+b-2 j}$ & 1 & $\overline{4}$ & 6 & 4 & 1 & \\
\hline$\beta_{i, a+b-(2 j+1}$ & $\overline{0}$ & 0 & 0 & 0 & 0 & \\
\hline \begin{tabular}{l||l|l|}
$\beta_{i, k}$ & 0 & 0 \\
\end{tabular} & 0 & & & & & for 3 \\
\hline
\end{tabular}

Next, let us consider the case of $a_{1}=2$. Then $S$ and $X$ satisfy (1.2) when $r \geq 6$ and $b \not \equiv 2\left(\bmod a_{2}\right)$. In these cases, we can calculate $\beta(X)$ completely by Theorem 3.2 and Propositions 4.1. In Section 5, we solve Problem $(\dagger)$ for the missing case where $r \geq 6$ and $b \equiv 2\left(\bmod a_{2}\right)$. In consequence, we solve Problem $(\dagger)$ completely when $a_{1}=2$ and $a_{2} \geq 3$. See Theorem 5.3 for details. In Example 5.4, we apply this result to the case of $S=S(2,3)$, and as in Theorem 1.2 and Theorem 1.3, we obtain an explicit description of $\beta(X)$ for every $X$ considered in Problem $(\dagger)$. It turns out that $\beta(X)$ has six different types.

Organization of the paper. In Section 2, we recall some definitions and basic facts. In Section 3, we prove Theorem 3.1 and Theorem 3.2, which are our main results in this paper. Section 4 is devoted to give a complete description of $\beta(E(r, s, t))$. In Section 5 , we apply our results in the previous sections to the cases where $a_{1}=1, a_{1}=2$ and $a_{1}=a_{2}$. Also we present some examples that illustrate how our results can be applied to specific cases. At the end of Section 5, we provide some examples which show that the statements in Theorem 3.1 and Theorem 3.2 are sharp.

Acknowledgement. The first named author was supported by Basic Science Research Program through the National Research Foundation of Korea(NRF) funded by the Ministry of Education(NRF-2017R1D1A1B03031438). The second named author was supported by the Korea Research Foundation Grant funded by the Korean Government 
(NRF-2018R1D1A1B07041336).

\section{Preliminaries}

In this section, we recall some definitions and basic facts.

Notation and Remark 2.1. Let $R:=\mathbb{k}\left[x_{0}, \ldots, x_{r}\right]$ be the homogeneous coordinate ring of the projective $r$-space $\mathbb{P}^{r}$ defined over an algebraically closed field $\mathbb{k}$ of arbitrary characteristic.

(1) For a non-zero finitely generated graded $R$-module $M$, the graded Betti numbers are defined by $\beta_{i, j}(M):=\operatorname{dim}_{\mathbb{k}} \operatorname{Tor}_{i}^{R}(M, \mathbb{k})_{i+j}$. The Betti table of $M$, denoted by $\beta(M)$, is the table whose entry in the $i$-th column and $j$-th row is $\beta_{i, j}(M)$. Throughout this paper, we present $\beta(M)$ as follows:

$\beta(M)=$\begin{tabular}{|c||c|c|c|c|c|c|c|}
\hline$i$ & 0 & 1 & $\cdots$ & $i$ & $\cdots$ & $r-1$ & $r$ \\
\hline$\vdots$ & $\vdots$ & $\vdots$ & $\ddots$ & $\vdots$ & $\ddots$ & $\vdots$ & $\vdots$ \\
\hline$\beta_{i, j}(M)$ & $\beta_{0, j}(M)$ & $\beta_{1, j}(M)$ & $\cdots$ & $\beta_{i, j}(M)$ & $\cdots$ & $\beta_{r-1, j}(M)$ & $\beta_{r, j}(M)$ \\
\hline$\vdots$ & $\vdots$ & $\vdots$ & $\ddots$ & $\vdots$ & $\ddots$ & $\vdots$ & $\vdots$ \\
\hline$\beta_{i, 1}(M)$ & $\beta_{0,1}(M)$ & $\beta_{1,1}(M)$ & $\cdots$ & $\beta_{i, 1}(M)$ & $\cdots$ & $\beta_{r-1,1}(M)$ & $\beta_{r, 1}(M)$ \\
\hline$\beta_{i, 0}(M)$ & $\beta_{0,0}(M)$ & $\beta_{1,0}(M)$ & $\cdots$ & $\beta_{i, 0}(M)$ & $\cdots$ & $\beta_{r-1,0}(M)$ & $\beta_{r, 0}(M)$ \\
\hline$\vdots$ & $\vdots$ & $\vdots$ & $\ddots$ & $\vdots$ & $\ddots$ & $\vdots$ & $\vdots$ \\
\hline
\end{tabular}

(2) Let $\mathbb{B}_{r}:=\bigoplus_{-\infty}^{\infty} \mathbb{Z}^{r+1}$ be the additive group of all tables with $r+1$ columns whose entries are integers. We regard $\beta(M)$ as an element of $\mathbb{B}_{r}$.

(3) Let $\ell \in \mathbb{Z}$ and $T \in \mathbb{B}_{r}$. Then we denote by $T[\ell]$ the table obtained by lifting $T$ up to $\ell$ rows. That is, the $(i, j)$-th entry of $T[\ell]$ is exactly equal to the $(i, j-\ell)$-th entry of $T$.

(4) For a closed subscheme $X \subset \mathbb{P}^{r}$, we will denote by $\beta(X)$ the Betti table of the homogeneous ideal $I(X)$ of $X$ as a graded $R$-module.

Let $r$ and $s$ be integers such that $r \geq 3$ and $1 \leq s \leq r$. Consider a (possibly degenerate) rational normal curve $S(s) \subset \mathbb{P}^{r}$ of degree $s$. For each integer $t \geq 2$, we denote by $\mathcal{F}_{t}$ the line bundle on $S(s)$ of degree $-t$. Also we define $E(r, s, t)$ as the graded $R$-module associated to $\mathcal{F}_{t}$. That is,

$$
E(r, s, t)=\bigoplus_{m \in \mathbb{Z}} H^{0}\left(\mathbb{P}^{r}, \mathcal{F}_{t} \otimes \mathcal{O}_{\mathbb{P}^{r}}(m)\right) .
$$

In Section 4 , we get a complete description of $\beta(E(r, s, t))$ for all $r, s$ and $t$. For details, see Proposition 4.1 .

We finish this section by investigating a few basic properties of $E(r, s, t)$. If $x$ is a real number, let $\lceil x\rceil$ denote the smallest integer $\geq x$.

Lemma 2.2. Let $r, s, t$ and $E(r, s, t)$ be as above. Then,

For any integer $n \in \mathbb{Z}$, it holds that

$$
E(r, s, t+n s) \cong E(r, s, t)(-n) \quad \text { and hence } \quad \beta(E(r, s, t+n s))=\beta(E(r, s, t))[n] .
$$




$$
\begin{aligned}
& \operatorname{reg}(E(r, s, t))=\left\lceil\frac{t-1}{s}\right\rceil+1 \\
& \beta_{i, j}(E(r, s, t))=0 \text { if } j \neq\left\lceil\frac{t-1}{s}\right\rceil,\left\lceil\frac{t-1}{s}\right\rceil+1 . \text { Furthermore, if } t \equiv 1(\bmod s), \text { then } \\
& \beta_{i, j}(E(r, s, t))=0 \text { if } j \neq\left\lceil\frac{t-1}{s}\right\rceil+1 .
\end{aligned}
$$

Proof. (1) The assertions come from the fact that $\mathcal{F}_{t+n s} \cong \mathcal{F}_{t} \otimes \mathcal{O}_{\mathbb{P}^{r}}(-n)$.

(2) Write $t=s \times u+p$ for some $2 \leq p \leq s+1$. Thus $u=\left\lceil\frac{t-1}{s}\right\rceil-1$. Then, by (1), we have

$$
\operatorname{reg}(E(r, s, t))=\operatorname{reg}(E(r, s, p))+\left\lceil\frac{t-1}{s}\right\rceil-1 .
$$

Also the line bundle $\mathcal{O}_{\mathbb{P}^{1}}(-p)$ on $S(s)$ is 2-regular as a coherent sheaf on $\mathbb{P}^{r}$. This completes the proof.

(3) It is obvious that $\beta_{i, j}(E(r, s, t))=0$ if $j>\operatorname{reg}(E(r, s, t))$. For the remaining cases, we recall that for every $n \in \mathbb{Z}$,

$$
E(r, s, t)_{n}=H^{0}\left(\mathbb{P}^{r}, \mathcal{F}_{t} \otimes \mathcal{O}_{\mathbb{P}^{r}}(n)\right) \cong H^{0}\left(\mathbb{P}^{1}, \mathcal{O}_{\mathbb{P}^{1}}(n s-t)\right) .
$$

Therefore $E(r, s, t)_{n}=0$ if and only if $n \leq \frac{t-1}{s}$. In particular, we get $E(r, s, t)_{n}=0$ if $n \leq \operatorname{reg}(E(r, s, t))-2$. This implies that $\beta_{i, j}(E(r, s, t))=0$ if $j \leq \operatorname{reg}(E(r, s, t))-2$. For the last statement, suppose that $t \equiv 1(\bmod s)$. Then it holds that

$$
\frac{t-1}{s}=\operatorname{reg}(E(r, s, t))-1 \text {. }
$$

Therefore $E(r, s, t)_{n}=0$ if $n \leq \operatorname{reg}(E(r, s, t))-1$. In particular, $\beta_{i, j}(E(r, s, t))=0$ if $j=\operatorname{reg}(E(r, s, t))-1$.

\section{Decomposition Theorems of $\beta(X)$}

Throughout this section, let $S=S\left(a_{1}, a_{2}\right) \subset \mathbb{P}^{r}$ be a smooth rational normal surface scroll and $X$ be an effective divisor of $S$ linearly equivalent to $a H+b F$ for some $a, b \in \mathbb{Z}$ such that either $a=0$ and $b>a_{2}$ or else $a \geq 1$ and $b \geq 2$. We denote by $C_{0}$ the minimal section $S\left(a_{1}\right)$ of $S$. Note that $C_{0}$ is linearly equivalent to $H-a_{2} F$.

The aim of this section is to prove two theorems about the decomposition of $\beta(X)$ into the sum of several Betti diagrams that are much simpler.

To state our results about $\beta(X)$, we need the integers $\delta=\delta(X), \epsilon=\epsilon(X)$ and $q_{\ell}=$ $q_{\ell}(X)$ for $1 \leq \ell \leq \delta$ which are defined in terms of $a_{1}, a_{2}, a$ and $b$ as

$\delta:=\left\lceil\frac{b-1}{a_{2}}\right\rceil, \quad \epsilon:=b-(\delta-1) a_{2} \quad$ and $\quad q_{\ell}=a_{1} a+b+\left(a_{1}-a_{2}\right)(\ell-1) \quad$ for $\quad 1 \leq \ell \leq \delta$.

Therefore the integer $\delta(X)$ can be regarded as a measure of how far $X$ is from the arithmetically Cohen-Macaulay property since a curve linearly equivalent to $X+\ell C_{0}$ is $\mathrm{ACM}$ if and only if $\ell=\delta$. Indeed, see [P2, Theorem 4.3] for the cases where $a \geq 1$ or $a=0$ and $b>a_{2}+1$. Also, if $a=0$ and $b=a_{2}+1$ then $\delta(X)=1$ and $X+C_{0}$ is a rational normal curve which is apparently arithmetically Cohen-Macaulay. Also $q_{\ell}(X)$ is the intersection number of $X+(\ell-1) C_{0}$ and $C_{0}$. 
Theorem 3.1. Suppose that $a_{2} \geq 2 a_{1}-1$. Then

$$
\beta(X)=\beta(S)+\beta(E(H+\epsilon F))[a+\delta-2]+\sum_{\ell=1}^{\delta-1} \beta\left(E\left(r, a_{1}, q_{\ell}\right)\right) .
$$

Theorem 3.2. Suppose that $a_{2} \geq 2 a_{1}-1$ and $a_{1}+1 \leq \epsilon \leq a_{2}+1$. Then

$$
\beta(X)=\beta(S)+\beta\left(E\left(r-1, r-1, a_{1}+\epsilon\right)\right)[a+\delta-1]+\sum_{\ell=1}^{\delta} \beta\left(E\left(r, a_{1}, q_{\ell}\right)\right) .
$$

The following proposition plays a cornerstone in proving the above two theorems.

Proposition 3.3. Let $X \equiv a H+b F$ be an effective divisor of $S$ satisfying (1.1) and let $Y$ be the scheme-theoretic union of $X$ and $C_{0}$. Also put $q:=a a_{1}+b$. Then

(1) $\operatorname{reg}(X)=\operatorname{reg}\left(E\left(r, a_{1}, q\right)\right)$.

(2) $\operatorname{reg}(X) \geq \operatorname{reg}(Y)$. Moreover, $\operatorname{reg}(X)>\operatorname{reg}(Y)$ in the following cases:

(i) $a_{1}+2 \leq b \leq a_{2}+1$;

(ii) $a_{2} \geq a_{1}+1, b \geq a_{2}+2$ and $b \equiv \gamma\left(\bmod a_{1}\right)$ for some $2 \leq \gamma \leq a_{2}-a_{1}+1$.

(3) Suppose that $X$ does not contain $C_{0}$ as a component. Then there is an exact sequence of graded $R$-modules

$$
0 \rightarrow I(Y) \rightarrow I(X) \rightarrow E\left(r, a_{1}, q\right) \rightarrow 0
$$

where $I(X)$ and $I(Y)$ are respectively the homogeneous ideals of $X$ and $Y$ in $R$. If $\operatorname{reg}(X)>\operatorname{reg}(Y)$ or $\operatorname{reg}(X)=\operatorname{reg}(Y)$ and $b \equiv 1\left(\bmod a_{1}\right)$, then

$$
\beta(X)=\beta(Y)+\beta\left(E\left(r, a_{1}, q\right)\right) .
$$

(5) The above (3.3) holds if one of the following conditions holds:

(i) $a_{1}+1 \leq b \leq a_{2}+1$;

(ii) $a_{2} \geq a_{1}, b \geq a_{2}+2$ and $b \equiv \gamma\left(\bmod a_{1}\right)$ for some $1 \leq \gamma \leq a_{2}-a_{1}+1$.

Proof. (1) By Lemma 2.2. (2), we get $\operatorname{reg}\left(E\left(r, a_{1}, q\right)\right)=\left\lceil\frac{q-1}{a_{1}}\right\rceil+1$. Also $\operatorname{reg}(X)=a+1+$ $\left\lceil\frac{b-1}{a_{1}}\right\rceil$ by $\left[\mathrm{P} 2\right.$, Theorem 4.3]. Therefore $\operatorname{reg}(X)=\operatorname{reg}\left(E\left(r, a_{1}, q\right)\right)$.

(2) Since $C_{0} \equiv H-a_{2} F$, the divisor class of $Y$ is equal to $(a+1) H+\left(b-a_{2}\right) F$. Thus we get

$$
\operatorname{reg}(Y)= \begin{cases}a+2 & \text { if } 2 \leq b \leq a_{2}+1, \text { and } \\ a+2+\left\lceil\frac{b-a_{2}-1}{a_{1}}\right\rceil & \text { if } b \geq a_{2}+2 .\end{cases}
$$

by [P2, Theorem 4.3].

When $2 \leq b \leq a_{2}+1$, we have

$$
\operatorname{reg}(X)-\operatorname{reg}(Y)=\left\lceil\frac{b-1}{a_{1}}\right\rceil-1 \geq 0 .
$$

In particular, $\operatorname{reg}(X)>\operatorname{reg}(Y)$ if and only if $a_{1}+2 \leq b \leq a_{2}+1$.

Now, suppose that $b \geq a_{2}+2$. Then

$$
\operatorname{reg}(X)-\operatorname{reg}(Y)=\left\lceil\frac{b-1}{a_{1}}\right\rceil-\left\lceil\frac{b-a_{2}-1}{a_{1}}\right\rceil-1
$$


Thus $\operatorname{reg}(X)=\operatorname{reg}(Y)$ if $a_{1}=a_{2}$. Also if $a_{2}>a_{1}$, then it holds that

$$
\left\lceil\frac{b-1}{a_{1}}\right\rceil \geq\left\lceil\frac{b-a_{2}-1}{a_{1}}\right\rceil+\left\lceil\frac{a_{2}}{a_{1}}\right\rceil-1 \geq\left\lceil\frac{b-a_{2}-1}{a_{1}}\right\rceil+1
$$

and hence $\operatorname{reg}(X) \geq \operatorname{reg}(Y)$. Moreover, if $a_{2} \geq 2 a_{1}$ or if $a_{1}+1 \leq a_{2} \leq 2 a_{1}-1$ and $b \equiv \gamma\left(\bmod a_{1}\right)$ for some $2 \leq \gamma \leq a_{2}-a_{1}+1$, then one can check that

$$
\left\lceil\frac{b-1}{a_{1}}\right\rceil-\left\lceil\frac{b-a_{2}-1}{a_{1}}\right\rceil \geq 2
$$

and hence $\operatorname{reg}(X)>\operatorname{reg}(Y)$.

(3) The ideal sheaf $\mathcal{I}_{Y}$ of $Y$ in $\mathbb{P}^{r}$ is equal to $\mathcal{I}_{X} \cap \mathcal{I}_{C_{0}}$ and hence the quotient $\mathcal{I}_{X} / \mathcal{I}_{Y}$ is isomorphic to $\mathcal{I}_{\Gamma / C_{0}}$ where $\Gamma$ is the scheme-theoretic intersection of $X$ and $C_{0}$. Thus we have the exact sequence

$$
0 \rightarrow \mathcal{I}_{Y} \rightarrow \mathcal{I}_{X} \rightarrow \mathcal{O}_{C_{0}}(-\Gamma) \rightarrow 0
$$

of coherent sheaves on $\mathbb{P}^{r}$. Note that the length of $\Gamma$ is equal to $q$. Thus the graded $R$ module associated to $\mathcal{O}_{C_{0}}(-\Gamma)$ is equal to $E\left(r, a_{1}, q\right)$. From (3.7), we get the cohomology long exact sequence

$$
0 \rightarrow H^{0}\left(\mathbb{P}^{r}, \mathcal{I}_{Y}(j)\right) \rightarrow H^{0}\left(\mathbb{P}^{r}, \mathcal{I}_{X}(j)\right) \stackrel{\varphi_{j}}{\rightarrow} E\left(r, a_{1}, q\right)_{j} \rightarrow H^{1}\left(\mathbb{P}^{r}, \mathcal{I}_{Y}(j)\right) \rightarrow \cdots
$$

for every $j \in \mathbb{Z}$. Thus it needs to check that $\varphi_{j}$ is always surjective. In the proof of Lemma 2.2.(3), it is shown that $E\left(r, a_{1}, q\right)_{n}=0$ for $n \leq \operatorname{reg}\left(E\left(r, a_{1}, q\right)\right)-2=\operatorname{reg}(X)-2$ by (1). Also, by (1) and (2), we get

$$
H^{1}\left(\mathbb{P}^{r}, \mathcal{I}_{Y}(j)\right)=0 \quad \text { for } \quad j \geq \operatorname{reg}(X)-1 .
$$

In consequence, it is shown that $\varphi_{j}$ is surjective for all $j \in \mathbb{Z}$.

(4) From (3), we get the long exact sequence

$$
\begin{gathered}
\cdots \rightarrow \operatorname{Tor}_{i+1}^{R}\left(E\left(r, a_{1}, q\right), \mathbb{k}\right)_{i+j} \rightarrow \operatorname{Tor}_{i}^{R}(I(Y), \mathbb{k})_{i+j} \rightarrow \operatorname{Tor}_{i}^{R}(I(X), \mathbb{k})_{i+j} \\
\rightarrow \operatorname{Tor}_{i}^{R}\left(E\left(r, a_{1}, q\right), \mathbb{k}\right)_{i+j} \rightarrow \operatorname{Tor}_{i-1}^{R}(I(Y), \mathbb{k})_{i+j} \rightarrow \cdots
\end{gathered}
$$

Thus it suffices to show that

$$
\operatorname{Tor}_{i}^{R}(I(X), \mathbb{k})_{i+j} \cong \operatorname{Tor}_{i}^{R}(I(Y), \mathbb{k})_{i+j} \bigoplus \operatorname{Tor}_{i}^{R}\left(E\left(r, a_{1}, q\right), \mathbb{k}\right)_{i+j}
$$

as $\mathbb{k}$-vector spaces for all $i \geq 0$ and $j \leq \operatorname{reg}(X)$.

Firstly, suppose that $\operatorname{reg}(X)>\operatorname{reg}(Y)$. When $j=\operatorname{reg}(X)$ we get

$$
\operatorname{Tor}_{i}^{R}(I(Y), \mathbb{k})_{i+j}=\operatorname{Tor}_{i-1}^{R}(I(Y), \mathbb{k})_{i+j}=0 .
$$

When $j=\operatorname{reg}(X)-1$ it follows by Lemma 2.2.(3) that

$$
\operatorname{Tor}_{i+1}^{R}\left(E\left(r, a_{1}, q\right), \mathbb{k}\right)_{i+j}=\operatorname{Tor}_{i-1}^{R}(I(Y), \mathbb{k})_{i+j}=0 .
$$

Also, when $j \leq \operatorname{reg}(X)-2$ we get

$$
\operatorname{Tor}_{i+1}^{R}\left(E\left(r, a_{1}, q\right), \mathbb{k}\right)_{i+j}=\operatorname{Tor}_{i}^{R}\left(E\left(r, a_{1}, q\right), \mathbb{k}\right)_{i+j}=0 .
$$

In consequence, (3.9) is verified if $\operatorname{reg}(X)>\operatorname{reg}(Y)$.

Now, suppose that $\operatorname{reg}(X)=\operatorname{reg}(Y)$ and $b \equiv 1\left(\bmod a_{1}\right)$. Then

$$
\operatorname{Tor}_{i+1}^{R}\left(E\left(r, a_{1}, q\right), \mathbb{k}\right)_{i+j}=0 \quad \text { for all } j \neq \operatorname{reg}\left(E\left(r, a_{1}, q\right)\right)
$$


by Lemma 2.2. (3). Therefore it holds that

$$
\operatorname{Tor}_{i+1}^{R}\left(E\left(r, a_{1}, q\right), \mathbb{k}\right)_{i+j}=\operatorname{Tor}_{i-1}^{R}(I(Y), \mathbb{k})_{i+j}=0 \quad \text { if } j=\operatorname{reg}(X)
$$

and

$$
\operatorname{Tor}_{i+1}^{R}\left(E\left(r, a_{1}, q\right), \mathbb{k}\right)_{i+j}=\operatorname{Tor}_{i}^{R}\left(E\left(r, a_{1}, q\right), \mathbb{k}\right)_{i+j}=0 \quad \text { if } j<\operatorname{reg}(X) .
$$

This completes the proof of (3.9) when $\operatorname{reg}(X)=\operatorname{reg}(Y)$ and $b \equiv 1\left(\bmod a_{1}\right)$.

(5) By (2) and (4), (3.3) holds if $(i)$ or (ii) in (2) holds. Thus it remains to consider the cases where either

(i) $b=a_{1}+1$ or

(ii) $a_{2} \geq a_{1}, b \geq a_{2}+2$ and $b \equiv 1\left(\bmod a_{1}\right)$.

In these cases, we have either $\operatorname{reg}(X)>\operatorname{reg}(Y)$ or else $\operatorname{reg}(X)=\operatorname{reg}(Y)$ and $b \equiv 1(\bmod$ $\left.a_{1}\right)$. Therefore (3.3) holds by (4).

Proof of Theorem 3.1. If $a \geq 1$, then the line bundle $\mathcal{O}_{S}(X)$ on $S$ is very ample and hence there exists a smooth irreducible curve, say $X^{\prime}$. Since $\beta(X)=\beta\left(X^{\prime}\right)$ by $[\mathrm{P} 2$, Proposition 4.1(1)], we may assume that $X$ does not contain $C_{0}$ as a component.

(a) We will prove our theorem by induction on $\delta$. When $\delta=1$, we need to show that

$$
\beta(X)=\beta(S)+\beta(E(H+\epsilon F))[a-1] .
$$

To this aim, let $Z=H+\epsilon F$ and consider the two short exact sequences

$$
0 \rightarrow \mathcal{I}_{S} \rightarrow \mathcal{I}_{X} \rightarrow \mathcal{O}_{S}(-X) \rightarrow 0
$$

and

$$
0 \rightarrow \mathcal{I}_{S} \rightarrow \mathcal{I}_{Z} \rightarrow \mathcal{O}_{S}(-Z) \rightarrow 0 .
$$

Then we have the following two short exact sequences of $R$-modules

$$
0 \rightarrow I(S) \rightarrow I(X) \rightarrow E(X) \rightarrow 0 \text { and } 0 \rightarrow I(S) \rightarrow I(Z) \rightarrow E(Z) \rightarrow 0
$$

where $E(X)$ (resp. $E(Z)$ ) denotes the graded $R$-module associated to $\mathcal{O}_{S}(-X)$ (resp. $\left.\mathcal{O}_{S}(-Z)\right)$. Since $X-Z \equiv(a-1) H$, it holds that

$$
E(X) \cong E(Z)(-a+1) \text { and hence } \beta(E(X))=\beta(E(Z))[a-1] .
$$

Also, by [P2, Proposition 3.2], it holds that $\beta(X)=\beta(S)+\beta(E(X))$. Consequently, we get

$$
\beta(X)=\beta(S)+\beta(E(X))=\beta(S)+\beta(E(Z))[a-1]=\beta(S)+\beta(E(H+\epsilon F))[a-1] .
$$

Now, suppose that $\delta>1$ and let $Y=X \cup C_{0}$. Note that since $a_{2} \geq 2 a_{1}-1$ we can apply Proposition 3.3. (5) to $X$ whenever $b \geq a_{2}+2$. Therefore we have

$$
\beta(X)=\beta(Y)+\beta\left(E\left(r, a_{1}, q_{1}\right)\right) .
$$

Observe that $\delta(Y)=\delta(X)-1, \epsilon(X)=\epsilon(Y)$ and $q_{\ell}(Y)=q_{\ell+1}(X)$ for $1 \leq \ell \leq \delta(Y)$. By induction hypothesis, we have

$$
\beta(Y)=\beta(S)+\beta(E(H+\epsilon F))[(a+1)+\delta(Y)-2]+\sum_{\ell=1}^{\delta(Y)-1} \beta\left(E\left(r, a_{1}, q_{\ell}(Y)\right)\right) .
$$

Now, the desired formula (3.1) comes by combining (3.10) and (3.11). 
Proof of Theorem 3.2. To obtain the formula (1.2), we focus on the term $\beta(E(H+\epsilon F))$ in (1.1). Let $M$ be an irreducible curve on $S$ linearly equivalent to $H+\epsilon F$. Thus

$$
\beta(M)=\beta(S)+\beta(E(H+\epsilon F))
$$

by [P2, Proposition 3.2]. Now, let $N$ be the scheme-theoretic union of $M$ and $C_{0}$. Then we can apply Proposition 3.3. (5.i) to our case since $a_{1}+1 \leq \epsilon \leq a_{2}+1$. That is,

$$
\beta(M)=\beta(N)+\beta\left(E\left(r, a_{1}, a_{1}+\epsilon\right)\right) .
$$

Therefore we get

$$
\beta(E(H+\epsilon F))=-\beta(S)+\beta(N)+\beta\left(E\left(r, a_{1}, a_{1}+\epsilon\right)\right) .
$$

Now, observe that $N \equiv 2 H+\left(\epsilon-a_{2}\right) F$ and hence $N \subset \mathbb{P}^{r}$ is arithmetically CohenMacaulay (cf. [P2, Theorem 4.3]. Let $\Gamma \subset \mathbb{P}^{r-1}$ be a general hyperplane section of $N$. Then $\Gamma$ is contained in $S(r-1)$ since $N$ is a divisor of the rational normal surface scroll $S$. Also $|\Gamma|=\operatorname{deg}(N)=2 a_{1}+a_{2}+\epsilon$. Therefore we get

$$
\beta(\Gamma)=\beta(S(r-1))+\beta\left(E\left(r-1, r-1,2 a_{1}+a_{2}+\epsilon\right)\right)
$$

by [P2, Proposition 3.2]. Since $\beta(\Gamma)=\beta(N), \beta(S(r-1))=\beta(S)$ and $r-1=a_{1}+a_{2}$, it follows that

$$
\beta(N)-\beta(S)=\beta\left(E\left(r-1, r-1,2 a_{1}+a_{2}+\epsilon\right)\right)=\beta\left(E\left(r-1, r-1, a_{1}+\epsilon\right)\right)[1] .
$$

In consequence, it is shown that

$$
\beta(E(H+\epsilon F))=\beta\left(E\left(r-1, r-1, a_{1}+\epsilon\right)\right)[1]+\beta\left(E\left(r, a_{1}, a_{1}+\epsilon\right)\right) .
$$

By using the definitions of $q_{\delta}$ and $\epsilon$, one can check that

$$
\beta\left(E\left(r, a_{1}, \epsilon+a_{1}\right)\right)[a+\delta-2]=\beta\left(E\left(r, a_{1}, q_{\delta}\right)\right) .
$$

Now, we get the desired formula (3.2) by combining (3.1), (3.13) and (3.14).

\section{The Graded Betti numbers of the module $E(r, s, t)$}

In this section, we calculate the Betti numbers of the graded R-module $E(r, s, t)$, which is defined to be the graded $R$-module associated to the line bundle $\mathcal{O}_{\mathbb{P}^{1}}(-t)$ on a rational normal curve $S(s)$ of degree $s$ in $\mathbb{P}^{r}$.

Proposition 4.1. Suppose that $t=p+\ell$ for some $2 \leq p \leq s+1$. Then $\beta_{i, j}(E(r, s, t))=0$ if $j \neq\left\lceil\frac{t-1}{s}\right\rceil,\left\lceil\frac{t-1}{s}\right\rceil+1$. Also

$$
\beta_{i, \ell+1}(E(r, s, t))=\sum_{k=0}^{s+1-p}(s+1-p-k)\left(\begin{array}{l}
s \\
k
\end{array}\right)\left(\begin{array}{l}
r-s \\
i-k
\end{array}\right)
$$

and

$$
\beta_{i, \ell+2}(E(r, s, t))=\sum_{k=s+2-p}^{i+1}(k+p-s-1)\left(\begin{array}{l}
s \\
k
\end{array}\right)\left(\begin{array}{c}
r-s \\
i+1-k
\end{array}\right) .
$$

We will give a proof of Proposition 4.1 at the end of this section.

In the following corollaries, we show how all the Betti tables in the right hand side of (3.2) in Theorem 3.2 can be obtained from Proposition 4.1. 
Corollary 4.2. Let $S \subset \mathbb{P}^{r}$ be a surface of minimal degree. Then reg $(S)=2$ and $\beta(S)$ is of the form

$$
\beta(S)=\begin{array}{|l||l|l|l|l|l|}
\hline \beta_{i, 2}(S) & \beta_{0,2}(S) & \beta_{1,2}(S) & \cdots & \beta_{r-1,2}(S) & \beta_{r, 2}(S) \\
\hline
\end{array}
$$

where $\beta_{i, 2}(S)=(i+1)\left(\begin{array}{c}r-1 \\ i+2\end{array}\right)$ for all $i \geq 0$.

Proof. Let $C \subset \mathbb{P}^{r-1}$ be a general hyperplane section of $S$. Since $S$ is arithmetically Cohen-Macaulay, it holds that $\beta(S)=\beta(C)$. Also the homogeneous coordinate ring $A_{C}$ of $C$ is equal to $E(r-1, r-1, r-1)(-1)$ since $C$ is projectively normal. Therefore we have

$$
\beta_{i, 2}(S)=\beta_{i, 2}(C)=\beta_{i+1,1}\left(A_{C}\right)=\beta_{i+1,2}(E(r-1, r-1, r-1))=(i+1)\left(\begin{array}{l}
r-1 \\
i+2
\end{array}\right)
$$

for all $i \geq 0$ by Proposition 4.1.

Corollary 4.3. Suppose that $2 \leq p \leq r$. Then $\beta(E(r-1, r-1, p))$ is of the form

$$
\beta(E(r-1, r-1, p))=\begin{array}{|l||c|c|c|c|c|}
\hline \beta_{i, 2} & \beta_{0,2} & \beta_{1,2} & \cdots & \beta_{r-1,2} & \beta_{r, 2} \\
\hline \beta_{i, 1} & \beta_{0,1} & \beta_{1,1} & \cdots & \beta_{r-1,1} & \beta_{r, 1} \\
\hline
\end{array}
$$

where

$$
\beta_{i, 1}= \begin{cases}(r-p-i)\left(\begin{array}{c}
r-1 \\
i
\end{array}\right) & \text { for } 0 \leq i \leq r-1-p \\
0 & \text { for } r-p \leq i\end{cases}
$$

and

$$
\beta_{i, 2}= \begin{cases}0 & \text { for } 0 \leq i \leq r-1-p \\
(i+1+p-r)\left(\begin{array}{l}
r-1 \\
i+1
\end{array}\right) & \text { for } r-p \leq i\end{cases}
$$

Proof. The desired formulas are directly proved by Proposition 4.1.

Corollary 4.4. $\beta(E(r, s, s+1))$ is of the form

$$
\beta(E(r, s, s+1))=\begin{array}{|l||l|l|l|l|l|}
\hline \beta_{i, 2} & \beta_{0,2} & \beta_{1,2} & \cdots & \beta_{r-1,2} & \beta_{r, 2} \\
\hline
\end{array}
$$

where $\beta_{i, 2}=s\left(\begin{array}{c}r-1 \\ i\end{array}\right)$ for all $i \geq 0$.

Proof. This comes immediately from Proposition 4.1.

Example 4.5. By Proposition 4.1 and Corollary 4.4, we obtain $\beta(E(r, 2,2))$ and $\beta(E(r, 2,3))$. More precisely, we have

$$
\beta(E(r, 2,2))=\begin{array}{|l||c|c|c|c|c|c|c|}
\hline \beta_{i, 2} & \beta_{0,2} & \beta_{1,2} & \cdots & \beta_{i, 2} & \cdots & \beta_{r-1,2} & \beta_{r, 2} \\
\hline \beta_{i, 1} & \beta_{0,1} & \beta_{1,1} & \cdots & \beta_{i, 1} & \cdots & \beta_{r-1,1} & \beta_{r, 1} \\
\hline
\end{array}
$$

where

$$
\beta_{i, 1}=\beta_{i, 1}(E(r, 2,2))=\left(\begin{array}{c}
r-2 \\
i
\end{array}\right) \quad \text { and } \quad \beta_{i, 2}=\beta_{i, 2}(E(r, 2,2))=\left(\begin{array}{c}
r-2 \\
i-1
\end{array}\right)
$$

and

$$
\beta(E(r, 2,3))=\begin{array}{|l||l|l|l|l|l|l|l|}
\hline \beta_{i, 2} & \beta_{0,2} & \beta_{1,2} & \cdots & \beta_{i, 2} & \cdots & \beta_{r-1,2} & \beta_{r, 2} \\
\hline
\end{array}
$$


where

$$
\beta_{i, 2}=\beta_{i, 2}(E(r, 2,3))=2\left(\begin{array}{c}
r-1 \\
i
\end{array}\right)
$$

Remark 4.6. By Proposition 4.1, we need to compute $a_{1}$ distinct tables for given $r$ and $a_{1}$. Among these $a_{1}$ tables, there is an interesting relation. Indeed, let $p$ and $p^{\prime}$ be two integers such that $2 \leq p \leq s$ and $p^{\prime}=s+2-p$. Then, by using Proposition 4.1, one can show that

$$
\beta_{i, 1}(E(r, s, p))=\beta_{r-1-i, 2}\left(E\left(r, s, p^{\prime}\right)\right) \quad \text { and } \quad \beta_{i, 2}(E(r, s, p))=\beta_{r-1-i, 1}\left(E\left(r, s, p^{\prime}\right)\right) .
$$

For example, consider the case $a_{1}=3$. Then $\beta(E(r, 3,4))$ comes from Corollary 4.4. Also Proposition 4.1 shows that $\beta(E(r, 3,2))$ is of the form

$$
\beta(E(r, 3,2))=\begin{array}{|l||c|c|c|c|c|c|c|}
\hline \beta_{i, 2} & \beta_{0,2} & \beta_{1,2} & \cdots & \beta_{i, 2} & \cdots & \beta_{r-1,2} & \beta_{r, 2} \\
\hline \beta_{i, 1} & \beta_{0,1} & \beta_{1,1} & \cdots & \beta_{i, 1} & \cdots & \beta_{r-1,1} & \beta_{r, 1} \\
\hline
\end{array}
$$

where

$$
\beta_{i, 1}=\beta_{i, 1}(E(r, 3,2))=2\left(\begin{array}{c}
r-2 \\
i
\end{array}\right)+\left(\begin{array}{c}
r-3 \\
i-1
\end{array}\right) \quad \text { and } \quad \beta_{i, 2}=\beta_{i, 2}(E(r, 3,2))=\left(\begin{array}{c}
r-3 \\
i-2
\end{array}\right) .
$$

Now, one can quickly obtain $\beta(E(r, 3,3))$ by applying (4.1) to $\beta(E(r, 3,2))$. Namely, we have

$$
\beta(E(r, 3,3))=\begin{array}{|l||l|l|l|l|l|l|l|}
\hline \beta_{i, 2} & \beta_{0,2} & \beta_{1,2} & \cdots & \beta_{i, 2} & \cdots & \beta_{r-1,2} & \beta_{r, 2} \\
\hline \beta_{i, 1} & \beta_{0,1} & \beta_{1,1} & \cdots & \beta_{i, 1} & \cdots & \beta_{r-1,1} & \beta_{r, 1} \\
\hline
\end{array}
$$

where

$$
\beta_{i, 1}=\beta_{i, 1}(E(r, 3,3))=\left(\begin{array}{c}
r-3 \\
i
\end{array}\right) \quad \text { and } \quad \beta_{i, 2}=\beta_{i, 2}(E(r, 3,3))=2\left(\begin{array}{c}
r-2 \\
i-1
\end{array}\right)+\left(\begin{array}{c}
r-3 \\
i-1
\end{array}\right) .
$$

Proof of Proposition 4.1. The first part comes from Lemma 2.2.(3).

For the remaining cases, note that $\beta(E(r, s, t))=\beta(E(r, s, p))[\ell]$ (cf. Lemma 2.2. (1)). Thus we consider the module $E(r, s, p)$ associated to the line bundle $\mathcal{L}:=\mathcal{O}_{\mathbb{P}^{1}}(-p)$ on $S(s)$. To determine $\beta_{i, j}(E(r, s, p))$ for $j=1$ and $j=2$, we use the Koszul cohomology exact sequence

$$
\begin{gathered}
0 \rightarrow \operatorname{Tor}_{i}^{R}(E(r, s, p), \mathbb{k})_{i+j} \rightarrow H^{1}\left(\mathbb{P}^{r}, \bigwedge^{i+1} \mathcal{M} \otimes \mathcal{L}(j-1)\right) \rightarrow \bigwedge^{i+1} V \otimes H^{1}\left(\mathbb{P}^{r}, \mathcal{L}(j-1)\right) \\
\rightarrow H^{1}\left(\mathbb{P}^{r}, \bigwedge^{i} \mathcal{M} \otimes \mathcal{L}(j)\right) \rightarrow H^{2}\left(\mathbb{P}^{r}, \bigwedge^{i+1} \mathcal{M} \otimes \mathcal{L}(j-1)\right) \rightarrow \cdots
\end{gathered}
$$

where $\mathcal{M}=\Omega_{\mathbb{P}^{r}}(1)$ and $V=H^{0}\left(\mathbb{P}^{r}, \mathcal{O}_{\mathbb{P}^{r}}(1)\right)$ (cf. [G, Theorem (1.b.4)] or [E, Theorem 5.8]). Note that the restriction of $\mathcal{M}$ to $S(s) \cong \mathbb{P}^{1}$ is isomorphic to $\mathcal{O}_{\mathbb{P}^{1}}(-1)^{\oplus s} \oplus \mathcal{O}_{\mathbb{P}^{1}}^{\oplus(r-s)}$. 
For $j=1$, we get the cohomology vanishing $H^{2}\left(\mathbb{P}^{r}, \bigwedge^{i+1} \mathcal{M} \otimes \mathcal{L}\right)=0$ since $\mathcal{L}$ is supported on the curve $S(s)$. Therefore it holds that

$$
\begin{aligned}
\beta_{i, 1}(E(r, s, p))= & \operatorname{dim}_{\mathbb{k}} \operatorname{Tor}_{i}^{R}(E(r, s, p), \mathbb{k})_{i+1} \\
= & h^{1}\left(\mathbb{P}^{1}, \bigwedge^{i+1}\left(\mathcal{O}_{\mathbb{P}^{1}}(-1)^{\oplus s} \oplus \mathcal{O}_{\mathbb{P}^{1}}^{\oplus(r-s)}\right)(-p)\right)-\left(\begin{array}{c}
r+1 \\
i+1
\end{array}\right) h^{1}\left(\mathbb{P}^{1}, \mathcal{O}_{\mathbb{P}^{1}}(-p)\right) \\
& +h^{1}\left(\mathbb{P}^{1}, \bigwedge^{i}\left(\mathcal{O}_{\mathbb{P}^{1}}(-1)^{\oplus s} \oplus \mathcal{O}_{\mathbb{P}^{1}}^{\oplus(r-s)}\right) \otimes \mathcal{O}_{\mathbb{P}^{1}}(s-p)\right) \\
= & \sum_{k=0}^{i+1}(k+p-1)\left(\begin{array}{c}
s \\
k
\end{array}\right)\left(\begin{array}{c}
r-s \\
i+1-k
\end{array}\right)-(p-1)\left(\begin{array}{c}
r+1 \\
i+1
\end{array}\right) \\
& \quad+\sum_{k=s+2-p}^{i}(k+p-s-1)\left(\begin{array}{c}
s \\
k
\end{array}\right)\left(\begin{array}{c}
r-s \\
i-k
\end{array}\right)
\end{aligned}
$$

Also one can check that

$$
\begin{aligned}
\sum_{k=0}^{i+1}(k+p-1)\left(\begin{array}{l}
s \\
k
\end{array}\right)\left(\begin{array}{c}
r-s \\
i+1-k
\end{array}\right) & =\sum_{k=0}^{i+1} k\left(\begin{array}{l}
s \\
k
\end{array}\right)\left(\begin{array}{c}
r-s \\
i+1-k
\end{array}\right)+(p-1) \sum_{k=0}^{i+1}\left(\begin{array}{l}
s \\
k
\end{array}\right)\left(\begin{array}{c}
r-s \\
i+1-k
\end{array}\right) \\
& =s\left(\begin{array}{c}
r-1 \\
i
\end{array}\right)+(p-1)\left(\begin{array}{c}
r \\
i+1
\end{array}\right)
\end{aligned}
$$

and

$$
\begin{aligned}
\sum_{k=s+2-p}^{i} & (k+p-s-1)\left(\begin{array}{l}
s \\
k
\end{array}\right)\left(\begin{array}{l}
r-s \\
i-k
\end{array}\right) \\
= & -\sum_{k=0}^{s+1-p}(k+p-s-1)\left(\begin{array}{l}
s \\
k
\end{array}\right)\left(\begin{array}{l}
r-s \\
i-k
\end{array}\right)+\sum_{k=0}^{i}(k+p-s-1)\left(\begin{array}{l}
s \\
k
\end{array}\right)\left(\begin{array}{l}
r-s \\
i-k
\end{array}\right) \\
= & \sum_{k=0}^{s+1-p}(s+1-p-k)\left(\begin{array}{l}
s \\
k
\end{array}\right)\left(\begin{array}{l}
r-s \\
i-k
\end{array}\right)+\sum_{k=0}^{i} k\left(\begin{array}{l}
s \\
k
\end{array}\right)\left(\begin{array}{l}
r-s \\
i-k
\end{array}\right) \\
& +(p-s-1) \sum_{k=0}^{i}\left(\begin{array}{l}
s \\
k
\end{array}\right)\left(\begin{array}{l}
r-s \\
i-k
\end{array}\right) \\
= & \sum_{k=0}^{s+1-p}(s+1-p-k)\left(\begin{array}{l}
s \\
k
\end{array}\right)\left(\begin{array}{l}
r-s \\
i-k
\end{array}\right)+s\left(\begin{array}{l}
r-1 \\
i-1
\end{array}\right)+(p-s-1)\left(\begin{array}{l}
r \\
i
\end{array}\right) .
\end{aligned}
$$

Thus we get the desired formula for $\beta_{i, 1}(E(r, s, p))$. 
For $j=2$, we have $H^{1}\left(\mathbb{P}^{r}, \mathcal{L} \otimes \mathcal{O}_{\mathbb{P}^{r}}(1)\right)=H^{1}\left(\mathbb{P}^{1}, \mathcal{O}_{\mathbb{P}^{1}}(s-p)\right)=0$ and hence

$$
\begin{aligned}
\beta_{i, 2}(E(r, s, p)) & =\operatorname{dim}_{\mathbb{k}} \operatorname{Tor}_{i}^{R}(E(r, s, p), \mathbb{k})_{i+2} \\
& =h^{1}\left(\mathbb{P}^{1}, \bigwedge^{i+1}\left(\mathcal{O}_{\mathbb{P}^{1}}(-1)^{\oplus s} \oplus \mathcal{O}_{\mathbb{P}^{1}}^{\oplus(r-s)}\right)(s-p)\right) \\
& =\sum_{k=s+2-p}^{i+1}(k+p-s-1)\left(\begin{array}{l}
s \\
k
\end{array}\right)\left(\begin{array}{c}
r-s \\
i+1-k
\end{array}\right) .
\end{aligned}
$$

This completes the proof of the formula for $\beta_{i, 2}(E(r, s, p))$.

\section{Computation of $\beta(X)$ For some Cases}

In this section, we apply Theorem 3.2 and Propositions 4.1 to the cases where $S$ is equal to $S(1, r-2)$ for some $r \geq 3, S(2, r-3)$ for some $r \geq 6$ and $S(c, c)$ for some $c \geq 1$. As a consequence, we solve Problem $(\dagger)$ when $a_{1}=1$ and when $a_{1}=2$ and $a_{2} \geq 3$.

When $a_{1}=1$, Theorem 3.2 implies the following

Theorem 5.1. Let $S$ be the smooth rational normal surface scroll $S(1, r-2)$ in $\mathbb{P}^{r}$ and $X$ be an effective divisor of $S$ linearly equivalent to $a H+b F$ where either $a=0$ and $b \geq r-1$ or else $a \geq 1$ and $b \geq 2$. Then

$\beta(X)=\beta(S)+\beta(E(r-1, r-1,1+\epsilon))[a+\delta-1]+\sum_{\ell=1}^{\delta} \beta(E(r, 1,2))[a+b-(r-1)(\ell-1)]$.

Proof. Observe that Theorem 3.2 is applicable to every $X$ if $a_{1}=1$ and $q_{\ell}$ is equal to $a+b-(r-3)(\ell-1)$. This completes the proof.

Remark 5.2. (1) In Theorem 5.1, the Betti tables $\beta(S), \beta(E(r-1, r-1,1+\epsilon))$ and $\beta(E(r, 1,2))$ are completely calculated in Corollary 4.2, Corollary 4.3 and Corollary 4.4 .

(2) By applying Theorem 5.1, we can reprove Theorem 1.1.

(3) When $r \geq 4$, the integers $q_{1}, q_{2}, \ldots, q_{\delta}$ decrease strictly. Thus, Theorem 5.1 shows that for fixed $\delta$ and $a+b, \beta(X)$ depends only on the table $\beta(E(r-1, r-1,1+\epsilon))$. Therefore there are $(r-2)$ different types of $\beta(X)$ since $\epsilon$ can take $(r-2)$ different values.

Proof of Theorem 1.2. By Theorem 5.1, we have

$$
\beta(X)=\beta(S)+\beta(E(3,3,1+\epsilon))[a+\delta-1]+\sum_{\ell=1}^{\delta} \beta(E(4,1,2))[a+b-\ell-1] .
$$

Note that $\epsilon=2$ if $b=2 \delta$ and $\epsilon=3$ if $b=2 \delta+1$. Thus the proof is completed by Remark 5.2. (1).

Proof of Theorem 1.3. By Theorem 5.1, we have

$$
\beta(X)=\beta(S)+\beta(E(4,4,1+\epsilon))[a+\delta-1]+\sum_{\ell=1}^{\delta} \beta(E(5,1,2))[a+b-2 \ell] .
$$


Note that $\epsilon=2$ if $b=3 \delta-1, \epsilon=3$ if $b=3 \delta$ and $\epsilon=4$ if $b=3 \delta+1$. Thus the proof is again completed by Remark 5.2.(1).

Next, we consider the case where $S$ is equal to $S(2, r-3)$ for some $r \geq 6$. Theorem 3.2 gives us the following

Theorem 5.3. Let $S$ be the smooth rational normal surface scroll $S(2, r-3)$ in $\mathbb{P}^{r}$ for some $r \geq 6$ and $X$ be an effective divisor of $S$ linearly equivalent to $a H+b F$ where either $a=0$ and $b \geq r-2$ or else $a \geq 1$ and $b \geq 2$. Then

(a) If $\epsilon=2$, then $\beta(X)$ is decomposed as

$$
\beta(X)=\beta(S)+\beta(E(H+2 F))[a+\delta-2]+\sum_{\ell=1}^{\delta-1} \beta(E(r, 2,2 a+b+(5-r)(\ell-1)))
$$

where $\beta(E(H+2 F))$ is of the form

$$
\beta(E(H+2 F))=\begin{array}{|l||l|l|l|l|l|}
\hline \beta_{i, 3} & \beta_{0,3} & \beta_{1,3} & \cdots & \beta_{r-1,3} & \beta_{r, 3} \\
\hline \beta_{i, 2} & \beta_{0,2} & \beta_{1,2} & \cdots & \beta_{r-1,2} & \beta_{r, 2} \\
\hline
\end{array}
$$

and

$$
\beta_{i, 2}= \begin{cases}\left(\begin{array}{c}
r-2 \\
i-2
\end{array}\right)-\left(\begin{array}{c}
r+1 \\
i+1
\end{array}\right)+(i+2)\left(\begin{array}{c}
r-1 \\
i+1
\end{array}\right) & \text { for } 0 \leq i \leq r-4, \\
0 & \text { for } r-3 \leq i \leq r-1,\end{cases}
$$

and

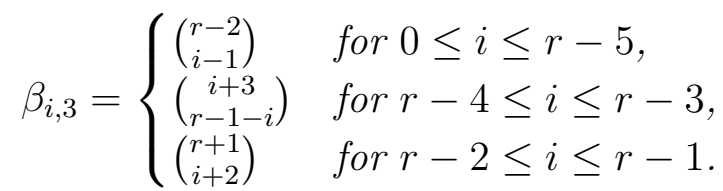

(b) If $3 \leq \epsilon \leq r-2$, then $\beta(X)$ is decomposed as

$\beta(X)=\beta(S)+\beta(E(r-1, r-1,2+\epsilon))[a+\delta-1]+\sum_{\ell=1}^{\delta} \beta(E(r, 2,2 a+b+(5-r)(\ell-1)))$.

Proof. One can check that $q_{\ell}=2 a+b+(5-r)(\ell-1)$. Thus the two decomposition formulas of $\beta(X)$ come immediately from Theorem 3.1 and Theorem 3.2, respectively. Thus it remains to show that $\beta(E(H+2 F))$ is equal to the one described above.

The line bundle $\mathcal{O}_{S}(H+2 F)$ is very ample and hence there is a smooth irreducible curve $\mathcal{C}$ on $S$ which is linearly equivalent to $H+2 F$. First we recall a geometric description of $\mathcal{C} \subset \mathbb{P}^{r}$ (cf. [P1, Theorem 1.1]). Since $\mathcal{C}$ is contained in $S=S(2, r-3)$ and $\operatorname{deg}(\mathcal{C})=r+1$, it holds that $\mathcal{C}=\pi_{P}(\widetilde{\mathcal{C}})$ where $\widetilde{\mathcal{C}} \subset \mathbb{P}^{r+1}$ is a rational normal curve of degree $r+1$ and $\pi_{P}: \widetilde{\mathcal{C}} \hookrightarrow \mathbb{P}^{r}$ is the isomorphic linear projection from a point $P \in \widetilde{\mathcal{C}}^{4} \backslash \widetilde{\mathcal{C}}^{3}$ where $\widetilde{\mathcal{C}}^{k}$ is the $k$-th join $\widetilde{\mathcal{C}}$ with itself. Thus it follows by [LP, Theorem 1.1] that

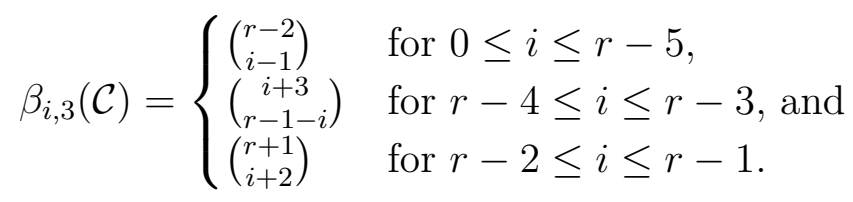


Also it holds by [Hoa, Theorem 2] that

$$
\beta_{i, 2}(\mathcal{C})= \begin{cases}\left(\begin{array}{l}
r \\
2
\end{array}\right)-2 & \text { for } i=0 \\
\beta_{i-1,3}(\mathcal{C})+r\left(\begin{array}{c}
r-1 \\
i+1
\end{array}\right)-\left(\begin{array}{c}
r-1 \\
i+2
\end{array}\right)-\left(\begin{array}{c}
r+1 \\
i+1
\end{array}\right) & \text { for } 1 \leq i \leq r-2, \text { and } \\
0 & \text { for } i=r-1 .\end{cases}
$$

By [P2, Proposition 3.2], we have $\beta(E(H+2 F))=\beta(\mathcal{C})-\beta(S)$. Therefore we get the desired description of $\beta(E(H+2 F))$ by combining Corollary 4.2, (5.1) and (5.2).

Example 5.4. Let $X$ be an effective divisor of $S=S(2,3)$ in $\mathbb{P}^{6}$ which is linearly equivalent to $a H+b F$ where either $a=0$ and $b \geq 4$ or else $a \geq 1$ and $b \geq 2$. Recall that

$$
\delta=\left\lceil\frac{b-1}{3}\right\rceil \text { and } \quad q_{\ell}=2 a+b+1-\ell \text { for all } 1 \leq \ell \leq \delta .
$$

Write $b=6 m+k$ for some $2 \leq k \leq 7$. For the simplicity, we denote $\beta(E(6,2,2))[a+2 m+t]$ and $\beta(E(6,2,3))[a+2 m+t]$ by $T_{2}(t)$ and $T_{3}(t)$, respectively. By Theorem $[5.3$, $\beta(X)$ can be decomposed into exactly one of the following six types according to the value of $b(\bmod 6)$.

Case 1. If $b=6 m+2$, then $\epsilon=2$ and $\beta(X)$ is decomposed as

$$
\beta(X)=\beta(S)+\beta(E(H+2 F))[a+2 m-1]+T_{3}(0)+\sum_{k=1}^{m-1}\left\{T_{2}(k)+T_{3}(k)\right\}+T_{2}(m)
$$

Case 2. If $b=6 m+3$, then $\epsilon=3$ and $\beta(X)$ is decomposed as

$$
\beta(X)=\beta(S)+\beta(E(5,5,5))[a+2 m]+T_{3}(0)+\sum_{k=1}^{m}\left\{T_{2}(k)+T_{3}(k)\right\}
$$

Case 3. If $b=6 m+4$, then $\epsilon=4$ and $\beta(X)$ is decomposed as

$$
\beta(X)=\beta(S)+\beta(E(5,5,6))[a+2 m]+\sum_{k=1}^{m}\left\{T_{2}(k)+T_{3}(k)\right\}+T_{2}(m+1)
$$

Case 4. If $b=6 m+5$, then $\epsilon=2$ and $\beta(X)$ is decomposed as

$$
\beta(X)=\beta(S)+\beta(E(H+2 F))[a+2 m]+T_{3}(1)+\sum_{k=1}^{m}\left\{T_{2}(k+1)+T_{3}(k+1)\right\}
$$

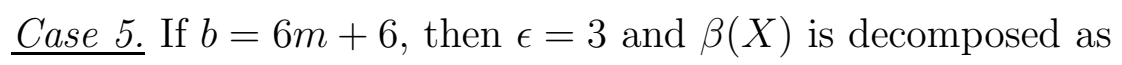

$$
\beta(X)=\beta(S)+\beta(E(5,5,5))[a+2 m+1]+T_{3}(1)+\sum_{k=1}^{m}\left\{T_{2}(k+1)+T_{3}(k+1)\right\}+T_{2}(m+2)
$$

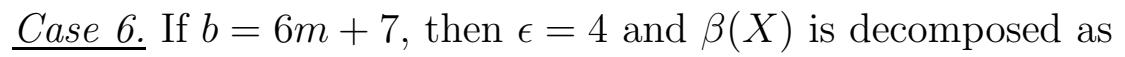

$$
\beta(X)=\beta(S)+\beta(E(5,5,6))[a+2 m+1]+\sum_{k=1}^{m+1}\left\{T_{2}(k+1)+T_{3}(k+1)\right\} .
$$


Also, using Corollary 4.2, Corollary 4.3, Example 4.5 and Theorem 5.3. (a), we can obtain $\beta(S), \beta(E(H+2 F)), \beta(E(5,5,5)), \beta(E(5,5,6)), \beta(E(6,6,2))$ and $\beta(E(6,6,3))$. The precise form of $\beta(X)$ for Case 1 - Case 6 are provided respectively in the following Table 3 and Table 4.

\begin{tabular}{|c||c|c|c|c|c|c||c|c|c|c|c|c||c|c|c|c|c|c|}
\hline$i$ & 1 & 2 & 3 & 4 & 5 & 6 & 1 & 2 & 3 & 4 & 5 & 6 & 1 & 2 & 3 & 4 & 5 & 6 \\
\hline \hline$\beta_{i, a+3 m+3}$ & 0 & 0 & 0 & 0 & 0 & 0 & 0 & 0 & 0 & 0 & 0 & 0 & 0 & 1 & 4 & 6 & 4 & 1 \\
\hline$\beta_{i, a+3 m+2}$ & 0 & 1 & 4 & 6 & 4 & 1 & 2 & 11 & 24 & 26 & 14 & 3 & 3 & 15 & 30 & 30 & 15 & 3 \\
\hline$\beta_{i, a+3 m+1}$ & 3 & 15 & 30 & 30 & 15 & 3 & 3 & 15 & 30 & 30 & 15 & 3 & 3 & 15 & 30 & 30 & 15 & 3 \\
\hline$\vdots$ & $\vdots$ & $\vdots$ & $\vdots$ & $\vdots$ & $\vdots$ & $\vdots$ & $\vdots$ & $\vdots$ & $\vdots$ & $\vdots$ & $\vdots$ & $\vdots$ & $\vdots$ & $\vdots$ & $\vdots$ & $\vdots$ & $\vdots$ & $\vdots$ \\
\hline$\beta_{i, a+2 m+3}$ & 3 & 15 & 30 & 30 & 15 & 3 & 3 & 15 & 30 & 30 & 15 & 3 & 3 & 15 & 30 & 30 & 15 & 3 \\
\hline$\beta_{i, a+2 m+2}$ & 3 & 15 & 36 & 39 & 18 & 3 & 3 & 24 & 46 & 39 & 15 & 2 & 6 & 24 & 36 & 24 & 6 & 0 \\
\hline$\beta_{i, a+2 m+1}$ & 3 & 9 & 6 & 0 & 0 & 0 & 1 & 0 & 0 & 0 & 0 & 0 & 0 & 0 & 0 & 0 & 0 & 0 \\
\hline$\beta_{i, a+2 m}$ & 0 & 0 & 0 & 0 & 0 & 0 & 0 & 0 & 0 & 0 & 0 & 0 & 0 & 0 & 0 & 0 & 0 & 0 \\
\hline$\vdots$ & $\vdots$ & $\vdots$ & $\vdots$ & $\vdots$ & $\vdots$ & $\vdots$ & $\vdots$ & $\vdots$ & $\vdots$ & $\vdots$ & $\vdots$ & $\vdots$ & $\vdots$ & $\vdots$ & $\vdots$ & $\vdots$ & $\vdots$ & $\vdots$ \\
\hline$\beta_{i, 3}$ & 0 & 0 & 0 & 0 & 0 & 0 & 0 & 0 & 0 & 0 & 0 & 0 & 0 & 0 & 0 & 0 & 0 & 0 \\
\hline$\beta_{i, 2}$ & 10 & 20 & 15 & 4 & 0 & 0 & 10 & 20 & 15 & 4 & 0 & 0 & 10 & 20 & 15 & 4 & 0 & 0 \\
\hline
\end{tabular}

TABLE $3 . b=6 m+2, b=6 m+3$ and $b=6 m+4$

\begin{tabular}{|c||c|c|c|c|c|c||c|c|c|c|c|c||c|c|c|c|c|c|}
\hline$i$ & 1 & 2 & 3 & 4 & 5 & 6 & 1 & 2 & 3 & 4 & 5 & 6 & 1 & 2 & 3 & 4 & 5 & 6 \\
\hline \hline$\beta_{i, a+3 m+4}$ & 0 & 0 & 0 & 0 & 0 & 0 & 0 & 1 & 4 & 6 & 4 & 1 & 2 & 11 & 24 & 26 & 14 & 3 \\
\hline$\beta_{i, a+3 m+3}$ & 2 & 11 & 24 & 26 & 14 & 3 & 3 & 15 & 30 & 30 & 15 & 3 & 3 & 15 & 30 & 30 & 15 & 3 \\
\hline$\beta_{i, a+3 m+2}$ & 3 & 15 & 30 & 30 & 15 & 3 & 3 & 15 & 30 & 30 & 15 & 3 & 3 & 15 & 30 & 30 & 15 & 3 \\
\hline$\vdots$ & $\vdots$ & $\vdots$ & $\vdots$ & $\vdots$ & $\vdots$ & $\vdots$ & $\vdots$ & $\vdots$ & $\vdots$ & $\vdots$ & $\vdots$ & $\vdots$ & $\vdots$ & $\vdots$ & $\vdots$ & $\vdots$ & $\vdots$ & $\vdots$ \\
\hline$\beta_{i, a+2 m+4}$ & 3 & 15 & 30 & 30 & 15 & 3 & 3 & 15 & 30 & 30 & 15 & 3 & 3 & 15 & 30 & 30 & 15 & 3 \\
\hline$\beta_{i, a+2 m+3}$ & 3 & 15 & 36 & 39 & 18 & 3 & 3 & 24 & 46 & 39 & 15 & 2 & 6 & 24 & 36 & 24 & 6 & 0 \\
\hline$\beta_{i, a+2 m+2}$ & 3 & 9 & 6 & 0 & 0 & 0 & 1 & 0 & 0 & 0 & 0 & 0 & 0 & 0 & 0 & 0 & 0 & 0 \\
\hline$\beta_{i, a+2 m+1}$ & 0 & 0 & 0 & 0 & 0 & 0 & 0 & 0 & 0 & 0 & 0 & 0 & 0 & 0 & 0 & 0 & 0 & 0 \\
\hline$\vdots$ & $\vdots$ & $\vdots$ & $\vdots$ & $\vdots$ & $\vdots$ & $\vdots$ & $\vdots$ & $\vdots$ & $\vdots$ & $\vdots$ & $\vdots$ & $\vdots$ & $\vdots$ & $\vdots$ & $\vdots$ & $\vdots$ & $\vdots$ & $\vdots$ \\
\hline$\beta_{i, 3}$ & 0 & 0 & 0 & 0 & 0 & 0 & 0 & 0 & 0 & 0 & 0 & 0 & 0 & 0 & 0 & 0 & 0 & 0 \\
\hline$\beta_{i, 2}$ & 10 & 20 & 15 & 4 & 0 & 0 & 10 & 20 & 15 & 4 & 0 & 0 & 10 & 20 & 15 & 4 & 0 & 0 \\
\hline
\end{tabular}

TABLE $4 . b=6 m+5, b=6 m+6$ and $b=6 m+7$

Here, the Betti numbers lying in the vertical dots on Table 3 and Table 4 are as follows:

\begin{tabular}{l||l|l|l|l|l|l|l|l|}
\hline$\beta_{i, a+j}$ & 3 & 15 & 30 & 30 & 15 & 3 & & for $\left\lceil\frac{b-1}{3}\right\rceil+2 \leq j \leq\left\lceil\frac{b-1}{3}\right\rceil+\left\lceil\frac{b-7}{6}\right\rceil$ and \\
\hline$\beta_{i, k}$ & 0 & 0 & 0 & 0 & 0 & 0 & & for $3 \leq k \leq a+\left\lceil\frac{b-4}{3}\right\rceil$
\end{tabular}

We finish this section by providing some examples which show that the hypotheses $a_{2} \geq 2 a_{1}-1$ and $a_{1}+1 \leq \epsilon \leq a_{2}+1$ in Theorem 3.2 can not be weakened. 
Example 5.5. Let $X$ be an effective divisor of $S=S(2,3)$ linearly equivalent to $H+11 F$. We have

$$
\delta(X)=4, \quad \epsilon(X)=2 \quad \text { and } \quad q_{\ell}(X)=14-\ell \quad \text { for } 1 \leq \ell \leq 4 .
$$

Theorem 5.3 says that $\beta(X)$ is decomposed as

$\beta(X)=\beta(S)+\beta(E(H+2 F))[3]+\beta(E(6,2,3))[4]+\beta(E(6,2,2))[5]+\beta(E(6,2,3))[5]$.

Now, by using Corollary 4.2, Example 4.5 and Theorem 5.3.(a), we can write $\beta(X)$ explicitly as below.

$\beta(X)=$\begin{tabular}{|c||c|c|c|c|c|c|}
\hline$\beta_{i, 7}$ & 2 & 11 & 24 & 26 & 14 & 3 \\
\hline$\beta_{i, 6}$ & 3 & 15 & 36 & 39 & 18 & 3 \\
\hline$\beta_{i, 5}$ & 3 & 9 & 6 & 0 & 0 & 0 \\
\hline$\beta_{i, 4}$ & 0 & 0 & 0 & 0 & 0 & 0 \\
\hline$\beta_{i, 3}$ & 0 & 0 & 0 & 0 & 0 & 0 \\
\hline$\beta_{i, 2}$ & 10 & 20 & 15 & 4 & 0 & 0 \\
\hline
\end{tabular}

From $\beta(X)$ in Example 5.5, we can see that the hypothesis $a_{1}+1 \leq \epsilon(X) \leq a_{2}+1$ in Theorem 3.2 cannot be weakened. Indeed, let $T$ denote the right hand side of (3.2) when $X$ is equal to the divisor $X$ of $S(2,3)$. That is,

$$
\begin{aligned}
T:= & \beta(S)+\beta(E(5,5,4))[4] \\
& +\beta(E(6,2,2))[4]+\beta(E(6,2,3))[4]+\beta(E(6,2,2))[5]+\beta(E(6,2,3))[5] .
\end{aligned}
$$

One can easily check that $\beta(X) \neq T$ and hence Theorem 3.2 fails to hold for $X$.

Finally, we consider some curves on the smooth rational normal surface scroll $S=$ $S(c, c)$ for some $c \geq 1$.

Theorem 5.6. Let $S=S(c, c) \subset \mathbb{P}^{2 c+1}$ be a smooth rational normal surface scroll and let $X$ be an effective divisor of $S$ linearly equivalent to $a H+(u c+1) F$ for some $a \geq 0$ and $u \geq 1$. Then $\beta(X)$ is of the form

$\beta(X)=$\begin{tabular}{|c||c|c|l|c|c|}
\hline$\beta_{i, a+u+1}$ & $\beta_{0, a+u+1}$ & $\beta_{1, a+u+1}$ & $\cdots$ & $\beta_{r-1, a+u+1}$ & $\beta_{r, a+u+1}$ \\
\hline$\beta_{i, a+u}$ & 0 & 0 & $\cdots$ & 0 & 0 \\
\hline$\vdots$ & $\vdots$ & $\vdots$ & $\ddots$ & $\vdots$ & $\vdots$ \\
\hline$\beta_{i, 3}$ & 0 & 0 & $\cdots$ & 0 & 0 \\
\hline$\beta_{i, 2}$ & $\beta_{0,2}$ & $\beta_{1,2}$ & $\cdots$ & $\beta_{r-1,2}$ & $\beta_{r, 2}$ \\
\hline
\end{tabular}

where

$$
\beta_{i, 2}=(i+1)\left(\begin{array}{c}
2 c \\
i+2
\end{array}\right) \quad \text { and } \quad \beta_{i, a+u+1}=(i+1)\left(\begin{array}{c}
2 c \\
i+1
\end{array}\right)+u c\left(\begin{array}{c}
2 c \\
i
\end{array}\right) \quad \text { for all } i \geq 0 .
$$

Proof. One can check that $\delta(X)=u, \epsilon(X)=c+1$ and $q_{\ell}(X)=(a+u) c+1$ for all $1 \leq \ell \leq u$. Thus, by using Proposition 3.3.(5) repeatedly, we obtain the decomposition

$$
\beta(X)=\beta(Z)+\sum_{\ell=1}^{u} \beta(E(2 c+1, c,(a+u) c+1))
$$


of $\beta(X)$ where $Z$ is an irreducible divisor of $S$ linearly equivalent to $(a+u) H+F$. Note that $Z \subset \mathbb{P}^{2 c+1}$ is arithmetically Cohen-Macaulay (cf. [P2, Theorem 4.3]). Now, let $\Gamma \subset \mathbb{P}^{2 c}$ be a general hyperplane section of $Z$. Thus we have

$$
\beta(Z)=\beta(\Gamma) \text { and }|\Gamma|=2 c(a+u)+1
$$

Also $\Gamma$ is contained in $S(2 c)$ since $Z$ is a divisor of $S$. By [ $\mathrm{P} 2$, Proposition 3.2], it follows that

$$
\beta(\Gamma)=\beta(S(2 c))+\beta(E(2 c, 2 c, 2 c(a+u)+1)) .
$$

In consequence, $\beta(X)$ is decomposed as

$$
\beta(X)=\beta(S)+\beta(E(2 c, 2 c, 2 c+1))[a+u-1]+u \times \beta(E(2 c+1, c, c+1))[a+u-1] .
$$

Thus we get the desired result by combining Corollary 4.3, Corollary 4.4 and (5.3) .

Remark 5.7. When $S$ is the smooth quadric $S(1,1)$ in $\mathbb{P}^{3}$, we can apply Theorem 5.6 to every effective divisor $X$ of $S$ linearly equivalent to $a H+b F$ for some $a \geq 0$ and $b \geq 2$. Therefore, Theorem 5.6 reproves Theorem 1.1 .

Example 5.8. Let $S=S(2,2)$ in $\mathbb{P}^{5}$ and $X$ be an effective divisor of $S$ linearly equivalent to $a H+b F$ where either $a=0$ and $b \geq 3$ or else $a \geq 1$ and $b \geq 2$.

(1) When $b=2 m+1$ for some $m \geq 1$, Theorem 5.6 shows that

$\beta(X)=$\begin{tabular}{|c||c|c|c|c|c|}
\hline$\beta_{i, a+m+1}$ & $b+3$ & $4 b+8$ & $6 b+6$ & $4 b$ & $b-1$ \\
\hline$\beta_{i, a+m}$ & 0 & 0 & 0 & 0 & 0 \\
\hline$\vdots$ & $\vdots$ & $\vdots$ & $\vdots$ & $\vdots$ & $\vdots$ \\
\hline$\beta_{i, 3}$ & 0 & 0 & 0 & 0 & 0 \\
\hline$\beta_{i, 2}$ & 6 & 8 & 3 & 0 & 0 \\
\hline
\end{tabular}

(2) Consider the case where $X \equiv H+4 F$. Thus $\delta(X)=\epsilon(X)=2$ and $q_{1}(X)=6$. Let $T$ denote the right hand side of (3.1) for this $X$. That is,

$$
T:=\beta(S)+\beta(E(H+2 F))[1]+\beta(E(5,2,2))[2] .
$$

Then we have

$$
T=\begin{array}{|c||c|c|c|c|c|}
\hline \beta_{i, 4} & 0 & 5 & 13 & 9 & 2 \\
\hline \beta_{i, 3} & 3 & 6 & 3 & 1 & 0 \\
\hline \beta_{i, 2} & 6 & 8 & 3 & 0 & 0 \\
\hline
\end{array} \quad \text { and } \quad \beta(X)=\begin{array}{|c||c|c|c|c|c|}
\hline \beta_{i, 4} & 0 & 5 & 12 & 9 & 2 \\
\hline \beta_{i, 3} & 3 & 6 & 3 & 0 & 0 \\
\hline \beta_{i, 2} & 6 & 8 & 3 & 0 & 0 \\
\hline
\end{array}
$$

where $\beta(X)$ is computed by means of the computer algebra system SINGULAR GP. In particular, $T \neq \beta(X)$. This example shows that the hypothesis $a_{2} \geq 2 a_{1}-1$ in Theorem 3.1 cannot be weakened.

\section{REFERENCES}

[EH] D. Eisenbud and J. Harris, On varieties of minimal degree (A centennial account), Proceedings of Symposia in Pure Mathematics 46 (1987), 3-13.

[E] D. Eisenbud, The Geometry of Syzygies, no.229, Springer-Velag New York, (2005)

[Fe] R. Ferraro, Weil divisors on rational normal scrolls, Lecture Notes in Pure and Applied Mathematics, 217 (2001), 183-198.

[GP] Gert-Martin Greuel, Gerhard Pfister et al Singular 3.0, a computer algebra system for polynomial computations, Center for Computer Algebra, University of Kaiserslautern (2005) (http://www.singular.uni-kl.de). 
[GM] S. Giuffrida and R. Maggioni, On the resolution of a curve lying on a smooth cubic surface in $\mathbb{P}^{3}$, Trans. Am. Math. Soc. 331 (1992), 181-201.

[G] M. Green, Koszul cohomology and the geometry of projective varieties, J. Differential Geom. 19 (1984), 125-171.

[H] J. Harris, Curves in projective space. With the collaboration of David Eisenbud, Seminaire de Mathematiques Superieures, 85. Presses de l'Universite de Montreal, Montreal, Que., (1982).

[Hoa] L. T. Hoa, On minimal free resolutions of projective varieties of degree=codimension+2, J. Pure Appl. Algebra 87 (1993), 241-250.

[LP] W. Lee and E. Park, Projective curves of degree=codimension+2 II, Int. J. Algebra Comput. Vol. 26, No. 1, (2016), 95-104.

[M] C. Miyazaki, Sharp bounds on Castelnuovo-Mumford regularity, Trans. Amer. Math. Soc. 352 (2000), no. 4, 1675-1686.

[MV] C. Miyazaki and W. Vogel, Bounds on cohomology and Castelnuovo-Mumford regularity, J. Algebra 185 (1996), no. 3, 626-642.

[N] U. Nagel, Arithmetically Buchsbaum divisors on varieties of minimal degree, Trans. Am. Math. Soc. 351, 4381-4409 (1999)

[P1] E. Park, Projective curves of degree = codimension+2, Math. Z. 256 (2007), no. 3, 685-697.

[P2] E. Park On syzygies of divisors of rational normal scrolls, Math. Nachr. 287 (2014), no. 11-12, $1383-1393$.

Department of Applied Mathematics, Pukyong National University, Busan 608-737, KOREA

E-mail address: wslee@pknu.ac.kr

Department of Mathematics, Korea University, Seoul 136-701, Korea

E-mail address: euisungpark@korea.ac.kr 